\title{
Psychiatrischer Beitrag
}

\section{Die Wirksamkeit psychologischer Interventionen in Gefängnissen auf die Rückfallraten nach der Entlassung}

\author{
J. Pellowski ${ }^{1}$ A. Voulgaris ${ }^{1}$ \\ Angenommen: 13. Dezember 2021 / Online publiziert: 5. Januar 2022 \\ (c) Der/die Autor(en) 2022
}

Personen, die aus Gefängnissen entlassen werden, haben ein höheres Risiko, erneut straffällig zu werden, als Personen, die anderweitig für Straftaten sanktioniert werden (Petersilia 2011). In Gefängnissen werden deshalb häufig psychotherapeutische Angebote gemacht, mit dem Ziel, die Rückfallraten der Insassen nach Entlassung sowie damit verbundene gesellschaftliche und gesundheitliche Kosten zu reduzieren. Dabei legen bisher veröffentlichte Reviews nahe, dass kognitiv-behaviorale Behandlungsprogramme zu den effektivsten Interventionen gehören; bisherige Metaanalysen verweisen auf eine Reduktion der Rückfälligkeit zwischen 20 und 30\% (z. B. Landenberger und Lipsey 2005). Außerdem zeigt sich ein Zusammenhang zwischen der Anwendung der „Risk-need-responsivity“-Prinzipien und einer reduzierten Rückfälligkeit.

Allerdings basieren die bisherigen Erkenntnisse vorwiegend auf quasiexperimentellen Studien, sodass die Befunde bezüglich der Wirksamkeit intramuraler Interventionen auf die Rückfälligkeit nach Entlassung insgesamt uneinheitlich und mit einer Reihe von Einschränkungen verbunden sind. So betrachten frühere Reviews spezifische Untersuchungsgruppen, es gibt erhebliche methodologische Unterschiede zwischen den Reviews, oder Wirksamkeitsschätzungen kombinieren unterschiedliche intramurale Umgebungen. Damit besteht die Gefahr, dass Wirksamkeitseffekte überschätzt werden.

Im Lancet Psychiatry veröffentlichen nun Beaudry et al. (2021) ein systematisches Review und eine Metaanalyse mit

\footnotetext{
J. Pellowski

j.pellowski@uke.de

$\triangle$ Dr. med. A. Voulgaris

a.voulgaris@uke.de

1 Universitätsklinikum Hamburg-Eppendorf, Hamburg,
} Deutschland dem Ziel, alle verfügbaren randomisierten kontrollierten Studien zusammenzufassen, die die Wirksamkeit intramuraler psychologischer Interventionen hinsichtlich der Rückfälligkeit nach Entlassung evaluieren. Bisherige Befunde sollen aktualisiert werden, einen breiteren Aussagebereich über spezifische Gruppen treffen und präzisere Aussagen liefern als frühere Reviews.

Die Einschlusskriterien wurden von 29 randomisierten kontrollierten Studien erfüllt. Ausgeschlossen wurden Studien, in denen einzig psychopharmakologische Interventionen durchgeführt wurden, die Studienteilnehmer:innen in psychiatrischen Gefängnis- oder sonstigen speziellen Wohneinheiten untergebracht waren oder die Insassen eine begleitende externe Therapie außerhalb des Gefängnisses wahrnahmen. Aus den geeigneten randomisierten kontrollierten Studien wurden Gesamtschätzungen entnommen und Effektgrößen in „odds ratios“ (OR) überführt. Um das Verzerrungspotenzial in den einzelnen Studien zu bewerten, orientierten sich die Autor:innen an einem Tool der Cochrane Collaboration (,risk-of-bias tool for randomized trials"). Das primäre Outcome war die Rückfälligkeit nach Entlassung. Die Daten einzelner randomisierter kontrollierter Studien wurden als gepoolte Odds ratios in eine „Random-effects"-Metaanalyse überführt und Quellen der Heterogenität $\left(I^{2}\right)$ untersucht, indem die Effektgrößen mit der Studiengröße, Kontrollgruppe und Interventionsart verglichen wurden.

Wurden alle 29 Studien berücksichtigt, zeigte sich ein Zusammenhang zwischen intramuralen psychologischen Interventionen und einer reduzierten Rückfälligkeit nach Entlassung (OR $=0,72 ; 95 \%-\mathrm{KI}=0,56-0,92)$ mit einer moderaten Heterogenität $\left(I^{2}=49 \%\right)$. Dieser Effekt war allerdings nicht mehr signifikant, nachdem kleinere Studien mit weniger als 50 Personen in der Interventionsgruppe ausgeschlossen wurden $(\mathrm{OR}=0,87 ; 95 \%-\mathrm{KI}=0,68-1,11$; $\left.I^{2}=54 \%\right)$. In der Literatur wird generell auf diesen ,small- 
study effect" hingewiesen und vor einer Überschätzung von Effekten gewarnt. Es ergaben sich somit Hinweise für einen Publikationsbias.

Weder bei kognitiv-behavioralen Interventionen $(\mathrm{OR}=$ 1,$\left.00 ; 95 \%-\mathrm{KI}=0,69-1,44 ; I^{2}=60 \%\right)$ noch bei psychoedukativen Interventionen $(\mathrm{OR}=1,11 ; 95 \%-\mathrm{KI}=0,38-3,20$; $\left.I^{2}=79 \%\right)$ zeigte sich ein Zusammenhang mit der Rückfälligkeit nach Entlassung. Zwei Studien legten hingegen einen Zusammenhang zwischen ,therapeutic community programms“ (a.e. therapeutische Nachsorgeprogramme) und Rückfälligkeit nach Entlassung nahe $(\mathrm{OR}=0,64$; $95 \%$ $\left.\mathrm{KI}=0,46-0,91 ; I^{2}=0 \%\right)$. Nur bei 2 von 29 Studien gab es nach Anwendung des Risk-of-bias tool for randomized trials ein geringes Verzerrungspotenzial; die häufigsten Beeinflussungen waren dabei auf den Prozess der Randomisierung sowie auf Abweichungen von der intendierten Intervention zurückzuführen.

In univariaten Analysen zeigte sich ein signifikanter $\mathrm{Zu}$ sammenhang zwischen geschlechtsspezifischen Interventionen und späterer reduzierter Rückfälligkeit $(\mathrm{OR}=0,67$; $95 \%-\mathrm{KI}=0,50-0,90)$. Darüber hinaus zeigten sich dagegen keine Zusammenhänge zwischen Studiencharakteristika, wie beispielsweise Erscheinungsjahr (vor 1990 vs. nach 1990), Studienort (USA vs. andernorts), Interventionsformat (Einzelsetting vs. Gruppensetting oder kombiniert), Alter (kontinuierlich) oder "follow-up time“ (kontinuierlich) und den Effektgrößen in Subgruppen.

Die Autor:innen diskutieren, dass sie damit in ihrem Review und ihrer Metaanalyse höchstens einen moderaten Effekt hinsichtlich der Wirksamkeit psychologischer Interventionen innerhalb des Justizvollzuges auf die Rückfälligkeit nach Entlassung nachweisen konnten. Die Effekte sind somit kleiner, als Expertenmeinungen zuvor angaben. Außerdem zeigt sich kein klarer Effekt für Programme, die auf kognitiv-behavioralen Interventionen basieren. Hier führen die Autor:innen als Erklärungen an, dass diese Interventionen möglicherweise in keiner Verbindung zu einer psychosozialen Unterstützung nach Entlassung stehen, oder dass die Interventionen ursprünglich für die Behandlung psychischer Probleme entwickelt wurden und nicht für berufliche, finanzielle oder unterkunftsbezogene Schwierigkeiten, die wiederum zu einem erhöhten Rückfallrisiko breitragen. Es könnte aber auch sein, dass die Effekte in früheren Studien auf die Kombination von randomisierten kontrollierten Studien mit weniger qualitativen Untersuchungen zurückzuführen sind. Dagegen zeigt sich ein Effekt von therapeutischen Nachsorgeangeboten, der allerdings nur auf 2 Studien beruht, in denen besonders Menschen mit Substanzkonsumstörungen untersucht wurden.

Die Autor:innen weisen nachvollziehbarerweise auf eine Reihe von Limitationen hin. Die untersuchten Studien wur- den nur in Ländern mit einem hohen Durchschnittseinkommen durchgeführt, und die Anzahl der geeigneten Studien war eher gering, was auf die praktischen sowie ethischen Herausforderungen, gleichzeitig jedoch auch auf die Notwendigkeit zukünftig hochqualitativer Untersuchungen in Gefängnissen hinweist. Gerade in dieser Untersuchungspopulation gibt es durch eine hohe Fluktuation auch hohe Drop-out-Raten, sodass meist kleine und selektive Stichproben vorliegen.

Es gibt eine Reihe von praktischen Implikationen. Zum einen sollten intramurale Interventionen immer auch die psychosozialen Bedürfnisse der später zu entlassenden Personen berücksichtigen. So unterstreichen die gefundenen Effekte die Wichtigkeit einer psychosozialen Nachsorge nach der Entlassung, um intramurale therapeutische Effekte zu stabilisieren. Daher sollten in zukünftiger Forschung solche psychologischen Interventionen priorisiert werden, die gefängnisbasierte und gesellschaftsbasierte Angebote kombinieren. Zum anderen sollten therapeutische Interventionen an die Zielpopulation adaptiert werden, um veränderbare Risikofaktoren, die spezifisch für eine hohe Rückfälligkeit sind, zu adressieren.

Funding Open Access funding enabled and organized by Projekt DEAL.

Interessenkonflikt J. Pellowski und A. Voulgaris geben an, dass kein Interessenkonflikt besteht.

Open Access Dieser Artikel wird unter der Creative Commons Namensnennung 4.0 International Lizenz veröffentlicht, welche die Nutzung, Vervielfältigung, Bearbeitung, Verbreitung und Wiedergabe in jeglichem Medium und Format erlaubt, sofern Sie den/die ursprünglichen Autor(en) und die Quelle ordnungsgemäß nennen, einen Link zur Creative Commons Lizenz beifügen und angeben, ob Änderungen vorgenommen wurden.

Die in diesem Artikel enthaltenen Bilder und sonstiges Drittmaterial unterliegen ebenfalls der genannten Creative Commons Lizenz, sofern sich aus der Abbildungslegende nichts anderes ergibt. Sofern das betreffende Material nicht unter der genannten Creative Commons Lizenz steht und die betreffende Handlung nicht nach gesetzlichen Vorschriften erlaubt ist, ist für die oben aufgeführten Weiterverwendungen des Materials die Einwilligung des jeweiligen Rechteinhabers einzuholen.

Weitere Details zur Lizenz entnehmen Sie bitte der Lizenzinformation auf http://creativecommons.org/licenses/by/4.0/deed.de.

\section{Literatur}

Beaudry G, Yu R, Perry AE, Fazel S (2021) Effectiveness of psychological interventions in prison to reduce recidivism: a systematic review and meta-analysis of randomised controlled trials. Lancet Psychiatry 2021(8):759-773

Landenberger NA, Lipsey MW (2005) The positive effects of cognitive-behavioral programs for offenders: a meta-analysis of factors associated with effective treatment. J Exp Criminol 2005(1):451-476

Petersilia J (2011) Beyond the prison bubble. Wilson Q 2011(35):50-55 